\title{
BMJ Open Web-based early intervention for children with motor difficulties aged 3-8 years old using multimodal rehabilitation (WECARE): protocol of a patient-centred pragmatic randomised trial of paediatric telerehabilitation to support families
}

Chantal Camden (10 ,1,2 Jill G Zwicker (1) ,3,4 Melanie Morin,, ${ }^{1,2}$ Tibor Schuster, $^{5}$ Melanie Couture, ${ }^{1,2}$ Thomas G Poder, ${ }^{6,7}$ Desiree B Maltais, ${ }^{8}$ Marie-Claude Battista, ${ }^{9}$ Jean-Patrice Baillargeon, ${ }^{2,10}$ Mathieu Goyette, ${ }^{11}$ Gabrielle Pratte, ${ }^{1}$ Karen Hurtubise, ${ }^{1}$ Michelle Phoenix, ${ }^{12}$ Tram Nguyen, ${ }^{13}$ Jade Berbari, ${ }^{2}$ Michel Tousignant, ${ }^{1}$ WECARE TEAM

To cite: Camden C, Zwicker JG, Morin M, et al. Web-based early intervention for children with motor difficulties aged 3-8 years old using multimodal rehabilitation (WECARE): protocol of a patient-centred pragmatic randomised trial of paediatric telerehabilitation to support families. BMJ Open 2021;11:e046561. doi:10.1136/ bmjopen-2020-046561

- Prepublication history and additional material for this paper are available online. To view these files, please visit the journal online (http://dx.doi. org/10.1136/bmjopen-2020046561).

Received 02 November 2020 Revised 07 December 2020 Accepted 15 March 2021

\section{Check for updates}

(C) Author(s) (or their employer(s)) 2021. Re-use permitted under CC BY-NC. No commercial re-use. See rights and permissions. Published by BMJ.

For numbered affiliations see end of article.

\section{Correspondence to}

Dr Chantal Camden; chantal.camden@usherbrooke. ca

\section{ABSTRACT}

Introduction Mild motor difficulties in children are underdiagnosed despite being highly prevalent, leaving such children often underserved and at higher risk for secondary consequences such as cardiovascular disease and anxiety. Evidence suggests that early patient-oriented interventions, coaching parents and providing children with early stimulation should be provided, even in the absence of a diagnosis. Such interventions may be effectively delivered via telerehabilitation.

Methods and analysis A family-centred, pragmatic randomised controlled trial will be carried out to evaluate the real-world effectiveness of a Web-based Early intervention for Children using multimodAl REhabilitation (WECARE). Families of children with motor difficulties, 3-8 years of age, living in Quebec, Canada, and receiving no public rehabilitation services $(n=118)$ will be asked to determine up to 12 performance goals, evaluated using the Canadian Occupational Performance Measure (COPM, the primary outcome). Families will be randomised to receive either usual care or the WECARE intervention. The WECARE intervention will be delivered for 1 year via a web-based platform. Families will have access to videoconferences with an assigned rehabilitation therapist using a collaborative coaching approach, a private chat function, a forum open to all intervention arm participants and online resources pertaining to child development. Participants will be asked to re-evaluate the child's COPM performance goals every 3 months up to 1 year post allocation. The COPM results will be analysed using a mixed Poisson regression model. Secondary outcomes include measures of the child's functional ability, parental knowledge and skills and healthrelated quality of life, as well as qualitative outcomes pertaining to parental satisfaction and service delivery trajectories. Investigators and quantitative data analysts will be blinded to group allocation.

Ethics and dissemination The CIUSSS de l'Estrie-CHUS ethics committee approved this trial (2020-3429). Study results will be communicated via peer-reviewed journal

\section{Strengths and limitations of this study}

The Web-based Early intervention for Children using multimodAl REhabilitation (WECARE) Study evaluates an innovative telerehabilitation intervention based on a multimodal web platform offering one-on-one therapist videoconferencing sessions, a private chat function, an online forum and online resources.

- This is the first randomised controlled trial where the families of children with mild motor difficulties are offered a telerehabilitation intervention.

- The WECARE Study takes a pragmatic and patientcentred approach, comparing the intervention results with usual care in a real-world setting with patient-centred goals as the primary outcome.

- WECARE aligns itself with current scientific evidence and new international paediatric rehabilitation recommendations for early intervention services to be non-categorical (ie, independent of the diagnosis), interdisciplinary, patient-centred and aimed at fostering childhood development and family empowerment.

- Results are based primarily on parents' perceptions and not on objective motor performance measures, which may limit study conclusions.

publications, conference presentations and stakeholderspecific knowledge transfer activities.

Trial registration number NCT04254302.

\section{INTRODUCTION}

Motor difficulties affect 400000-800000 Canadian children, ${ }^{12}$ half of whom do not have timely access to rehabilitation services 
to support their development. ${ }^{3}$ Motor difficulties are defined as having fine and/or gross motor skills below expectations for the child's age. ${ }^{4}$ Early interventions and rehabilitation are effective for improving children's health, development and functioning. ${ }^{5-7}$ However, only children with severe motor difficulties have access to these services, while children with so-called mild disabilities rarely receive timely services, facing waiting periods of up to 2 years. ${ }^{89}$ Children who experience these 'mild' disabilities, such as developmental coordination disorder (DCD; 5\%-6\% prevalence), are not only underserved but also often underdiagnosed, ${ }^{10}{ }^{11}$ further limiting access to services. ${ }^{12}$

Children with motor difficulties are at higher risk of cardiovascular disease, obesity, lower self-efficacy, social isolation, depression and anxiety. ${ }^{8-15}$ Motor difficulties and their secondary consequences also impact the children's family, who often report a lower sense of well-being and higher levels of stress, ${ }^{1617}$ an increased economic burden due to frequent medical consultations and care requirements ${ }^{17-22}$ and 'shopping for diagnoses and services' to gain access to specialised services. ${ }^{18} 19$ New evidence suggests that early prediagnosis patient-oriented interventions aimed at coaching parents and providing children with early stimulation should be provided even in the absence of a diagnosis. ${ }^{120-22}$ Healthcare systems have been relatively slow to adopt new evidence on how to best organise services. Numerous systematic reviews have documented the effectiveness of early intervention on childhood development. ${ }^{12} 2324$ These reviews and many editorials highlight the importance of stimulating children ${ }^{2526}$ and providing parental guidance to optimise development, functioning, health and well-being. ${ }^{27} 28$

Parental guidance to stimulate the child's development can be provided through telerehabilitation, defined as providing rehabilitation services at distance. ${ }^{29}$ Telerehabilitation can increase access to services and flexibility of care. ${ }^{30} 31$ A pilot study conducted by our team confirmed the relevance and feasibility of conducting a trial to evaluate web-based, early rehabilitation services for children with mild motor difficulties. ${ }^{32}{ }^{33}$ A systematic review exclusively examining paediatric telerehabilitation identified the same key approaches: child developmental stimulation and parental guidance. ${ }^{29}$ This systematic review confirmed that telerehabilitation interventions were effective for a broad range of childhood outcomes, including motor functioning, and suggested that multimodal technological approaches might be most effective. Results from a pilot study suggest that a web-based rehabilitation intervention is relevant for children with mild motor disabilities and is perceived to be particularly helpful in the early stages, when initial concerns arise about motor development. ${ }^{32}{ }^{33}$ These findings illustrated the need to develop a more comprehensive family-centred multimodal online intervention to support families of children with motor disabilities, the Web-based Early intervention for Children using multimodAl REhabilitation (WECARE) intervention. Children aged 3-8 years old are a key group, as so-called 'mild' motor difficulties often become apparent after the age of three and during the first years of school, when motor expectations increase.

\section{Research questions and objectives}

Can a multimodal early online rehabilitation improve the motor performance of 3-8year-old children with motor difficulties in comparison with usual care? The overarching goal of this family-centred, mixed-method, randomised, innovative pragmatic trial is to evaluate the effectiveness of the WECARE intervention for children aged 3-8 years with motor difficulties living in Quebec (Canada). The primary objective is to evaluate, in comparison with usual care, the effectiveness of the WECARE Programme for improving the child's performance on parent-identified motor targets.

Secondary objectives are as follows:

1. To evaluate the effect of the intervention on children's functional abilities, parental knowledge and skills and the children and parents' health-related quality of life (HRQoL).

2. To explore factors influencing the implementation of the intervention within the current service delivery context.

3. To compare the service delivery trajectories and costs across trial arms.

The primary hypothesis is that children in the experimental arm will perform better on motor performance outcomes than children in the usual care arm. Secondary hypotheses are that children in the experimental arm will display higher functional abilities, and their families will report greater knowledge and skills and and greater HRQoL, will use less public and private healthcare services and will incur fewer out-of-pocket and systemlevel expenses after the 1-year intervention period.

\section{METHODS AND ANALYSIS \\ Design}

A family-centred innovative pragmatic, parallel-group, allocation-concealed, superiority randomised controlled trial assessing real-world effectiveness of the WECARE intervention will be offered to families of children with motor difficulties living in Quebec, Canada, and receiving no public rehabilitation services. The parent or legal guardian of the child will be the primary respondent, but the entire family will have access to the WECARE intervention. A mixed-method parallel design ${ }^{34}$ will be used to concurrently collect quantitative and qualitative data.

This trial was designed according to the latest guidelines on pragmatic randomised controlled trials, ${ }^{35}{ }^{36}$ using the gold standard Standard Protocol Items: Recommendations for Interventional Trials (SPIRIT) guidelines (https://www.spirit-statement.org/) and the TElehealth in CHronic Disease (TECH) conceptual framework. The TECH model ${ }^{37}$ is a reference for structuring the design and evaluation of telehealth programmes to ensure interventions are acceptable to patients and delivered in a 


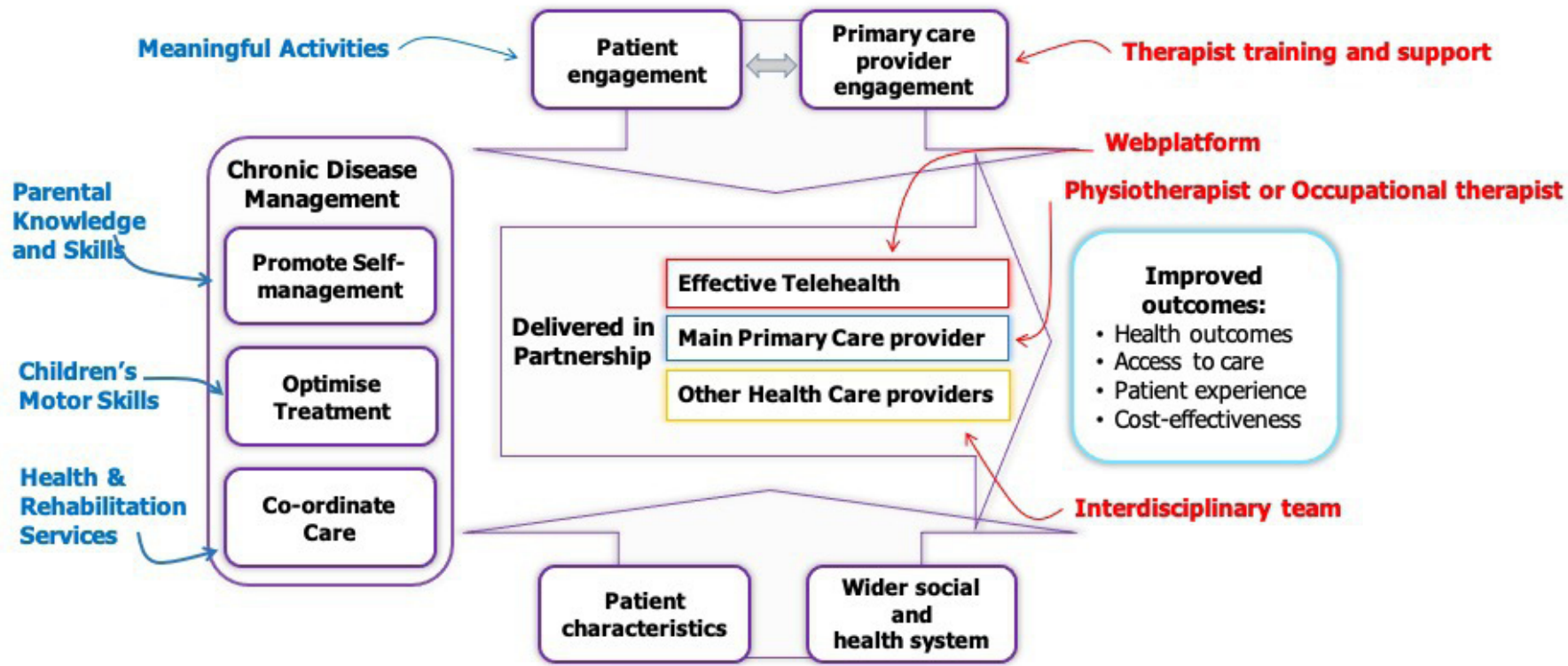

Figure 1 Illustration of how the Web-based Early intervention for Children using multimodAI REhabilitation (WECARE) intervention fits within the TElehealth in CHronic Disease $(\mathrm{TECH})$ model. The key active ingredients of the WECARE intervention are embedded into the patient engagement (ie, meaningful activity) and chronic management best practices (ie, parental knowledge and skills, children's motor skills and access to rehabilitation) of the TECH model and how the implementation processes build on therapist engagement (ie, training and support) and effective service delivery (ie, effective web platform, a primary therapist and an interdisciplinary team) to achieve the desired outcomes. Key ingredients are defined as the factors contributing directly to the expected outcomes, which are, in our case, known modifiable factors influencing children's motor development. A multimodal approach with various activities to deliver the key ingredients will be used. The implementation processes are defined as the mechanisms used to implement the intervention and activities into a real-world setting.

cost-effective manner. Figure 1 presents how the WECARE intervention fits within the TECH model, including its key active intervention ingredients.

\section{Intervention}

Control arm

Participants randomised into the control arm will receive usual care as per the service delivery models available in their region. Usual care may include referral for services, general recommendations or references to online content deemed appropriate by a healthcare professional or no services at all. As this is a pragmatic trial, no attempt will be made to standardise practices in the control arm.

\section{Experimental arm}

The WECARE intervention was developed based on lessons learnt in a previous pilot study, ${ }^{32} 33$ a systematic review of telerehabilitation interventions ${ }^{29}$ and a consultation of parents, clinicians and other stakeholders who may take part in such an intervention. It provides families of children with motor difficulties with access to rehabilitation therapists for 1 year. This duration was selected to reflect the sometimes seasonal aspect of motor difficulties, to capture whether changes in motor performance would occur without the WECARE intervention (eg, as the child ages or on access to services associated with long wait times) and to aid in the analysis of economic outcomes. The primary therapist will be an occupational therapist or a physiotherapist (herein 'therapist') working according to a transdisciplinary model. ${ }^{38}$ Therapists will use a collaborative coaching approach $^{4041}$ to support the family in helping the child achieve meaningful motor outcomes based on intervention objectives selected by the primary respondent. The active ingredients of the WECARE intervention will be delivered via a multimodal web-based platform, using a flexible approach, which is structured at first but adaptable and needs-based throughout:

Thirty-minute videoconference appointments with the therapist to help increase parental knowledge and skills in problem-solving for the child's motor performance issues, provide recommendations to support the child's motor development and assess and intervene online directly with the child, if needed; semimonthly appointments will be offered during the first 3 months and then on a participant-identified needs basis until the end of the intervention period.

Always available online resources with flexible access based on participant needs:

- A chat function where participants can privately contact the therapist to discuss concerns about the child's motor development.

- A forum open to all participants in the experimental arm where interparticipant and participanttherapist communication is fostered and participants 
are encouraged to ask questions and share their experiences.

- Static online information via links to relevant websites and other online child development resources.

The therapists may also act as case managers to connect families with community-based services available in their region. All therapists receive a 1-day training prior to their involvement in the study. They participate in weekly 30-minute transdisciplinary meetings to discuss ongoing cases with fellow therapists and monthly 60-minute mentoring and review meetings with the principal investigator.

\section{Sample size}

The analysis of the primary study outcome will be conducted using a mixed Poisson regression model that will estimate the difference in the attainment of clinically relevant performance goals between study arms (see the Primary outcome section). Extensive Monte Carlo simulations were conducted to determine the statistical power needed to detect differences in success rates between study groups. The chosen mixed Poisson model approach allows for a robust estimation of statistical power as important variance parameters (ie, within-arm SD) are implicitly determined by the expected success rates in the study arms. To enable a conservative sample size estimation, a relatively high baseline (control arm) success rate was assumed as well as a moderate number of predefined targets per study individual: the expected number of targets set at T0 per individual ranges from 1 to 12 , conservatively assuming a uniform distribution. A further conservative assumption was made that up to $25 \%$ of the defined targets may be achieved under control conditions and that heterogeneity in success rates across covariates may explain up to $10 \%$ of the total variation of the observed successes. Based on these cautious assumptions and a total of 100000 Monte Carlo simulations, it is expected that a sample size of 100 individuals (50 per arm) is sufficient to detect clinically relevant differences of $\geq 15$ percentage points in the success rate, that is, an increase from $25 \%$ to $40 \%$ (relative increase: 1.6 ) with more than $80 \%$ power. A retention rate of $85 \%$ was used, given the loss to follow-up rate reported for web-based interventions, ${ }^{42}$ the follow-up rate found in the feasibility trial $^{32}$ and the new adherence strategies included in this trial. This results in a targeted study sample of 59 participants per study arm, for a total of 118 participants.

\section{Study sample and recruitment}

Participants will be referred to the project by a health or education professional. They may also be self-referred (ie, will be recruited independently from any professional referral, via recruitment campaigns). Recruitment materials will contain a link to the online eligibility questionnaire, which the families will be invited to complete, should they, healthcare professionals or educators suspect that the child has motor difficulties. Selection bias will be minimised by working with a wide range of community, educational and healthcare partners and by using traditional recruitment methods (eg, email campaigns and print/digital media) and social media to ensure that recruitment activities reach the broadest possible population.

\section{Inclusion/exclusion criteria}

Participants will be the parent or legal guardian of a child: 1. Aged 3-8 years old.

2. With or at risk of motor difficulties, as confirmed via the DCD Questionnaire (DCDQ) or Little DCDQ.

3 . With at least one motor-related intervention objective prior to allocation, as confirmed via the Canadian Occupational Performance Measure (COPM).

4. Living in the province of Quebec, Canada.

5. With functional communication skills in French.

Participants will be excluded if they are receiving public physiotherapy or occupational therapy services for their motor difficulties at time of study enrolment. Eligibility criteria are inclusive and are not limited to children with an existing diagnosis related to motor difficulties so as to increase generalisability of the findings ${ }^{36}$ as they relate to all children with mild motor difficulties who do not have access to public rehabilitation services at time of recruitment.

\section{Eligibility process}

The eligibility questionnaire will give introductory information about the study, confirm geographical and agebased eligibility and ask the parent to complete an online version of the DCDQ or the Little DCDQ two validated questionnaires identifying subtle motor difficulties for children aged 3-4 and 5-8, respectively. ${ }^{43}{ }^{44}$ Eligible participants will be asked to consent online (please refer to the online supplemental file 1 to view the informed consent form) to participate in the full study; those who do so will be contacted by a member of the research team to schedule an online interview with a research assistant (T0 assessment). During this interview, the research assistant will confirm informed consent and eligibility criteria, including the meaningfulness and relevance of the child's motor difficulties via the COPM, ${ }^{45}$ as detailed below. On confirming their full eligibility, participants will be randomised into the experimental or control arm and will be granted access to the full intervention as appropriate.

\section{Allocation and blinding}

The family will be the unit of randomisation. A web-based randomisation service (REDCap) will allocate participants to either usual care or the WECARE intervention (1:1). To ensure equal distribution of participants between arms, permutated blocks of variable size will be generated. No stratification or minimisation strategies will be used.

Participants will set their motor performance goals prior to allocation to avoid the influence of the allocation arm on goals selection. Once allocation has occurred, 


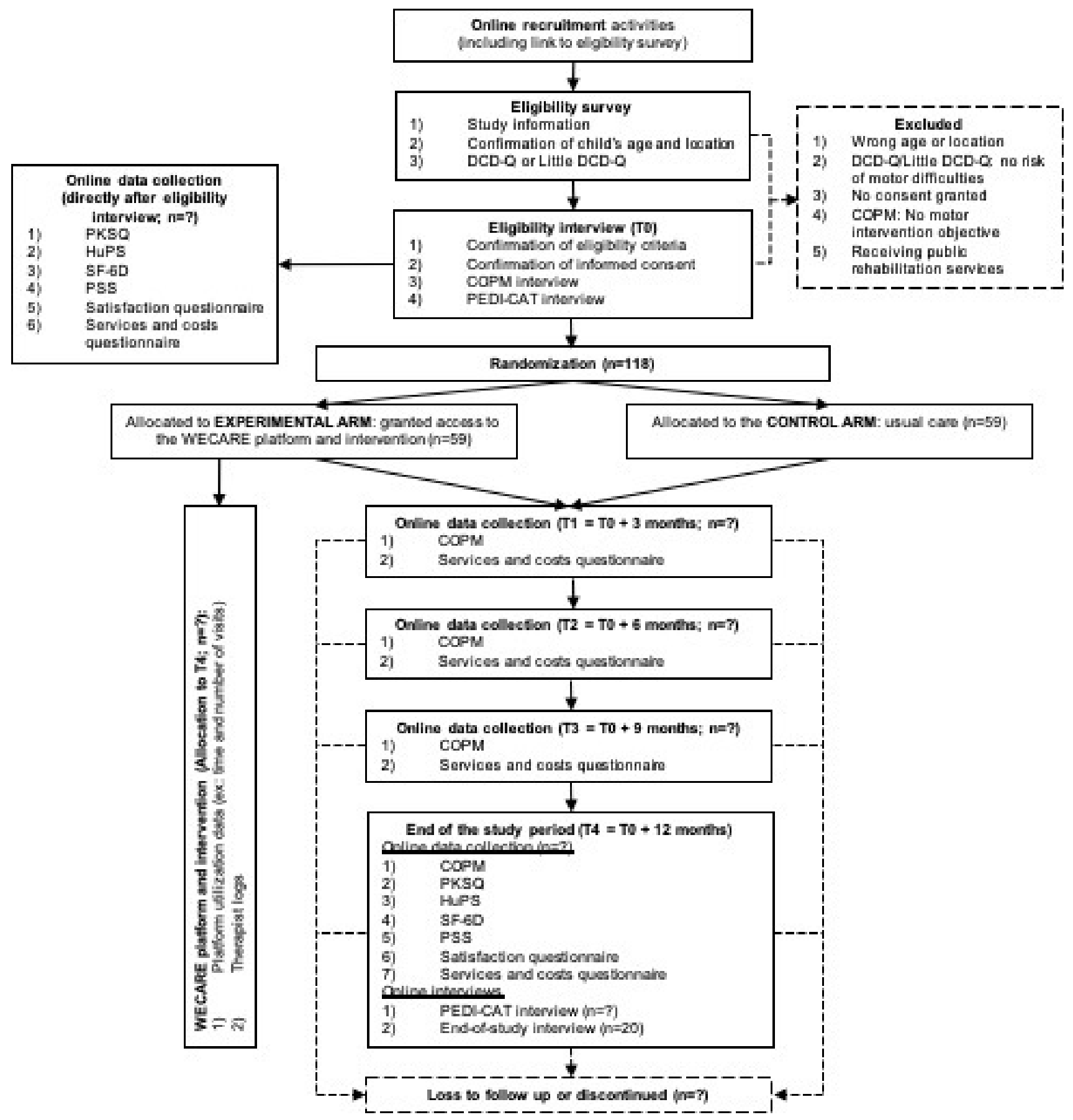

Figure 2 Patient timeline, eligibility process and data collection for the Web-based Early intervention for Children using multimodAI REhabilitation (WECARE) Study. COPM, Canadian Occupational Performance Measure; (Little) DCDQ, (Little) Developmental Coordination Disorder Questionnaire; HuPS, Health-Related Quality of Life Utility Measure for Preschool Children; PEDI-CAT, Paediatric Evaluation of Disability Inventory-Computer Adaptive Test; PKSQ, Parental Knowledge and Skills Questionnaire; PSS, Parental Stress Scale; SF-6D, short-form six dimensions.

the participant, the study coordinator and the therapists will be aware of participant allocation. Investigators and quantitative data analysts will be blinded to group allocation; no unblinding procedures are expected.

\section{Outcomes and data collection}

The participant timeline is presented in figure 2, including the screening, eligibility and data collection processes.

\section{Primary outcome}

The child's performance on motor goals and the parent's satisfaction with their child's performance, as well as the importance of the goals, will be collected with the COPM. The COPM uses a structured interview to identify intervention goals. Ten-point Visual Analogue Scales (VAS) are used to identify the importance, present performance and satisfaction with performance for 
each selected goal. Higher scores indicate higher importance, better performance and higher satisfaction with the child's performance. ${ }^{46}$ An increase of more than two points on the 10-point VAS is considered as a meaningful clinical difference ${ }^{47}$ and will be used as an indication of the attainment of the child's motor performance goal. The COPM does not require any physical or hands-on materials ${ }^{45}$ which is consistent with an online trial. The COPM is a clinical and research tool ${ }^{48}$ providing information about the impact of the intervention on activities and participation. ${ }^{47}$ It is the best tool available to measure changes in a child's performance for child-specific items not easily assessed with other standardised instruments. ${ }^{46}$ Its validity, reliability and sensitivity to change have been extensively studied. ${ }^{48}$ A published paediatric adaptation of the COPM will be used in this trial. ${ }^{47}$ The paediatric adaptation of the COPM has been validated with parents of children between 2 and 8 years old. ${ }^{46}{ }^{47}$ It showed good construct and criterion validity and inter-rater agreement. ${ }^{46}$ A French version of the COPM has been used with parents ${ }^{49}$ and to evaluate web-based interventions for children with disabilities. ${ }^{50}$

\section{Primary outcome data collection}

At T0, a research assistant will conduct an online interview where participants will be asked to identify up to 12 motor performance-related goals and will be asked to rate each goal on the performance, satisfaction and importance COPM scales. They will then be asked to select up to three primary goals to inform the intervention for the following 3-month period. This will ensure identification of all goals before participants are aware of their allocation arm. Every 3 months, participants will self-complete online the three COPM scales for each goal, select their main goals for the next 3-month period and identify new goals if applicable. For participants in the experimental arm, therapists will be informed of these goals. For the primary analysis, only the attainment of goals identified at $\mathrm{T} 0$ will be considered, based on performance scores. For the initial goals attained, secondary analyses will document the magnitude of change (ie, using raw VAS performance score) and the time to achieve goals (ie, months elapsed to achieve a 2-point or more increase), as well as the changes in importance and satisfaction scores, between arms.

\section{Secondary outcomes instruments and data collection}

All secondary outcome measures are validated and have previously been used with children with motor difficulties, use continuous data, are sensitive to change and are available in French. All secondary outcome measures can be completed and submitted online by the participants, with the exception of the Paediatric Evaluation of Disability Inventory-Computer Adaptive Test (PEDI-CAT), which requires an online interview with a research assistant. Secondary outcome measures will only be collected at $\mathrm{T} 0$ and $\mathrm{T} 4$.
Changes in children's functional abilities will be evaluated with the mobility and self-care subscales of the PEDI-CAT. ${ }^{51}$ The PEDI-CAT is a parent-reported adaptative questionnaire for parents of children and youth aged $0-20$ years. ${ }^{51}$ Each question is selected by the computer algorithm based on the child's socio-demographic data and answers to previous questions, up to a maximum of 15 for each domain. ${ }^{51}$ The number of items needed to establish the child's score may vary according to the stopping rules of the algorithm. ${ }^{51}$ For each question, parents indicate their child's ability to participate in a presented activity (eg, putting on socks) on a 4-point scale ranging from 'unable=can't do, doesn't know how or is too young' to 'easy=does with no help, extra time, or effort, or child's skills are past this level'. ${ }^{52}$ Subscale scores are expressed as T-scores. Content validity analysis confirmed each domain is unidimensional. ${ }^{52}$ All domains of the PEDI-CAT have excellent test-retest reliability. ${ }^{53}$

Changes in the parent's knowledge and skills about motor difficulties will be evaluated via the French version of the Parental Knowledge and Skills Questionnaire (PKSQ) ${ }^{54}$ The PKSQ has been validated for parents of children with DCD aged 0-18years old. ${ }^{54}$ An adapted version of the PKSQ will be used to remove the focus from DCD and ensure its relevance for all motor difficulties. The adapted PKSQ includes two out of the three constructs of the original version of the PKSQ. The adapted version includes 14 questions about parental understanding of motor difficulties and their perception of their capacity to manage their child's motor difficulties. Questions are scored by the parent on a 7-point Likert Scale ranging from 'not at all' to 'to a very great extent'. ${ }^{54}$

Parents will rate children's HRQoL with the HealthRelated Quality of Life Utility Measure for Preschool Children (HuPS) tool, an HRQoL tool for children aged 2-5 years. ${ }^{55}$ The HuPS includes 12 questions about the child's abilities rated on 3-point to 5-point scales (eg, seeing, speaking, using hand and fingers and self-care). Intrarater and inter-rater reliability is good. ${ }^{55}$

Changes in the parent's HRQoL will be measured with the short-form six dimensions (SF-6D) ${ }^{56}$ The SF-6D is a tool measuring health utility in adults and includes items on physical and social functioning, role limitations, pain, mental health and vitality. Total scores range from -0.574 to 1 (worse possible health to perfect health). The minimally important differences for SF-6D range from 0.011 to 0.097 , with a mean of $0.041 .^{56}$ This tool has previously been used to describe HRQoL among parents of children with disabilities. ${ }^{57}$

Parental stress will be measured with the Parental Stress Scale (PSS), consisting of 18 questions about parental feelings and experience. ${ }^{58}$ Each statement is scored on a 5-point Likert Scale (1=strongly agree to $5=$ strongly disagree). Higher scores indicate lower parental stress. The PSS has a good concurrent validity and test-retest reliability. ${ }^{58}$ 
Satisfaction, service delivery trajectories, health economics and platform utilisation measures

Satisfaction with the intervention will be evaluated via questionnaires adapted from Dunst, Trivette and Hamby $^{59}$; open-ended questions; and interview guides. The use of public rehabilitation services during the study will be documented for participants in both arms, as this reflects how this intervention would be rolled out in real life (ie, WECARE may be offered before face-toface publicly funded interventions but might overlap with them). Service delivery trajectories as well as systemlevel and out-of-pocket costs will be documented every 3 months via a questionnaire about services received in the last 3 months (ie, public or private healthcare and school-based services). The use of the platform by participants in the experimental arm will be assessed in terms of the number of visits and the time spent on the WECARE platform overall and in each subsection (ie, videoconferencing, private chat, forum and resources), as well as the number of chat messages sent to the therapist, the number of new threads or posts created on the forum and the number of resources consulted.

\section{Data analysis}

Analyses will be conducted according to the intentionto-treat principle. The primary outcome will be analysed using a mixed Poisson regression model, as the dependent variable consists of count data and observations are independent, to determine if families in the experimental arm attained more clinically meaningful goals than the families in the control arm. However, as a Poisson model assumes that mean and variance are identical, in the case where overdispersion would be observed, a negativebinomial regression model will be used. ${ }^{6061}$ The model will include, as offset variable, the number of individually defined targets and the following covariates: the severity of the child's motor difficulties (dichotomised as 'mild difficulties' or 'low functioning' based on PEDI-CAT T0 scores), as well as child and familial characteristics, that is, sex, age and income. Data will be reported as the exponentiated values of the coefficients of the mixed Poisson regression along with $95 \%$ CIs.

The effects of the WECARE intervention will also be examined within the following participant subgroups: severity of motor difficulties and child/participant sex. The effect of the following intervention-specific covariates will also be explored: intervention dosage (exposure to the different WECARE modes of service, ie, time spent on information, forums and chat and virtual appointments) and date of inclusion in the intervention (categorised by month).

Linear mixed-effect models will be used to compare secondary outcomes, expressed as continuous pre-post intervention scores. Participants will be treated as random effect, and an autocorrelation structure will be considered on repeated measures made on the same participant. The integration of quantitative and qualitative data will be conducted following an explanatory approach ${ }^{34}$ to provide a deeper understanding of the intervention effects according to variability in participant characteristics and the interactions with implementation processes.

Satisfaction, service delivery trajectories, health economics and platform utilisation measures

Information pertaining to satisfaction collected via questionnaires and interviews, including user acceptability of the WECARE intervention, will be analysed, respectively, with descriptive statistics and a mixed inductive-deductive qualitative approach of content analysis, ${ }^{62}$ building on the models used to conduct the interviews. ${ }^{37}$ The economic analysis will be based on incremental costeffectiveness ratios, ${ }^{63}$ using a $1.5 \%$ annual discount rate, and sensitivity analyses with discount rates of $0 \%$ and $3 \% .{ }^{64}$ Non-parametric analyses with 5000 bootstrap replications ${ }^{6566}$ will be performed to estimate the CI, while cost-effectiveness acceptability curves will compare on a probabilistic basis the cost-effectiveness thresholds for different costs per unit gain. ${ }^{67} 68$ Service delivery trajectories and platform utilisation measures, including the occurrence of technical issues, will be analysed with descriptive statistics. No interim analyses are planned for this study.

\section{Adherence}

All participants will be contacted every 3 months to encourage study adherence, coinciding with key data collection time points. For participants in the experimental arm, regular appointments with the therapist, every 2 weeks for the first 3 months, will foster additional adherence to the WECARE intervention. Furthermore, a notification system tied to a personal email will inform the participant of relevant content added to the WECARE platform, including new videoconferencing appointments and new communications sent by the therapist via the private chat function. To optimise adherence among participants in the control arm and to thank them for participating in the study, a 3-month access to the online forum and the resources section of the platform will be granted following the 1-year data collection period.

\section{Patient involvement}

Three patient partners (MJF, KT and CW) aided in the design of the WECARE intervention and of trial procedures, participated in the recruitment process and will contribute to knowledge translation activities.

\section{ETHICS AND DISSEMINATION}

No specific risk was reported in a recently conducted systematic review of telerehabilitation studies. ${ }^{29}$ Exercises or manipulations that may be undertaken as part of the intervention will target common daily activities and will be performed under parental supervision. Minor accidents (eg, falls during balance exercises) will be documented in a therapist logbook. Should therapists detect severe health conditions (eg, muscular dystrophy) or signs of 
distress (eg, suicidal ideation), they are instructed to refer to a physician or local resources identified by the research team. Adverse events will be reported, within 24 hours, to the executive committee (CC, MM, M-CB and JB). The executive committee will convene monthly to oversee all aspects of trial management. Given the digital nature of the intervention and data collection, the security of study data, be it for transit or storage, was at the forefront during the development of the web and data collection platforms. All data collection is undertaken via REDCap, a secured data collection platform hosted on University of Sherbrooke servers. The WECARE platform was developed with reputable partners (National Research Council of Canada) and is hosted on secure servers at the University of Sherbrooke. Due to the overall safety profile of this trial, no data and safety monitoring board was convened, and no interim analysis or stopping guidelines are expected. Evidence suggests that health inequity risks exist where wealthier families might benefit more from web-based interventions. ${ }^{51}$ Should eligible families have no internet access at home, the research team will work with community resources to provide participants with internet access. Such challenges will be documented to inform future trials.

The WECARE trial was registered at ClinicalTrials.gov and was approved by the ethics committee of the CIUSSS de l'Estrie-CHUS (identifier: 2020-3429). The ethics committee will be informed of any deviation from the protocol. No deviation will be applied until the amended protocol has been approved by the ethics committee. The executive committee will oversee the intrastudy and external data sharing process on a case by case basis; deidentified participant data as well as additional study documentation could be made available on reasonable request. Publications of study findings will be done via peer-reviewed journals and conference presentations, as well as stakeholder-specific knowledge transfer activities. Substantive contributions will be recognised in the authorship of these publications, as determined by the executive committee.

\section{PROJECT STATUS}

Recruitment began in March 2020 and ended in October 2020. Data collection is expected to end in October 2021, with the project closing in April 2022.

\section{Author affiliations}

1École de réadaptation, Universite de Sherbrooke Faculté de Médecine et des Sciences de la Santé, Sherbrooke, Québec, Canada

${ }^{2}$ Centre de recherche du Centre hospitalier universitaire de Sherbrooke, CIUSSS de I'Estrie-CHUS, Sherbrooke, Québec, Canada

${ }^{3}$ Occupational Science \& Occupational Therapy, The University of British Columbia, Vancouver, British Columbia, Canada

${ }^{4} \mathrm{BC}$ Children's Hospital, Vancouver, British Columbia, Canada

${ }^{5}$ Department of Family Medicine, McGill University, Montreal, Québec, Canada

${ }^{6}$ School of Public Health, Université de Montréal, Montreal, Québec, Canada

${ }^{7}$ Centre de recherche de l'Institut universitaire en santé mentale de Montréal,

Montreal, Québec, Canada

${ }^{8}$ Département de réadaptation, Universite Laval, Québec City, Québec, Canada
${ }^{9}$ Department of Medicine, Universite de Sherbrooke Faculté de Médecine et des Sciences de la Santé, Sherbrooke, Quebec, Canada

${ }^{10}$ Universite de Sherbrooke Faculté de Médecine et des Sciences de la Santé,

Sherbrooke, Quebec, Canada

${ }^{11}$ Département de sexologie, Université du Québec à Montréal, Montreal, Québec, Canada

${ }^{12}$ School of Rehabilitation Science, McMaster University, Hamilton, Ontario, Canada ${ }^{13}$ School of Epidemiology \& Public Health, University of Ottawa, Ottawa, Ontario, Canada

\section{Twitter Jill G Zwicker @jillzwicker}

Acknowledgements The research team would like to thank the following collaborators, who are all part of the WECARE team, for their contribution to study design and for reviewing the study protocol: Pasquale Roberge, Emmanuelle Jasmin, Mélissa Généreux, Julie Lane and Geneviève Corriveau of the Université de Sherbrooke, Sherbrooke, Canada; France Légaré of the Université Laval, Quebec, Canada; Audrée-Jeanne Beaudoin of the Institut Universitaire en DI et en TSA, Quebec, Canada; Kimberly Miller of the Sunny Hill Health Centre for Children, Vancouver, Canada; Deidre Speery and Olaf Kraus de Camargo of McMaster University, Hamilton, Canada; Lesley Pritchard-Wiart of the University of Alberta, Edmonton, Alberta, Canada; Holly Duchow and Sylvia Schell of Alberta Health Services, Central Zone, Camrose, Canada; Laura Bernard of Alberta Health Services, Edmonton, Canada; Sara Izadi-Najafabadi of the University of British Columbia, Vancouver, Canada; and Marie-Josée Fortier, Catherine Wilhelmy and Karine Tremblay of the Centre de recherche du Centre hospitalier universitaire de Sherbrooke, Sherbrooke, Canada.

Collaborators Pasquale Roberge, Emmanuelle Jasmin, Mélissa Généreux, Julie Lane, Geneviève Corriveau, France Légaré, Audrée-Jeanne Beaudoin, Kimberly Miller, Deidre Speery, Olaf Kraus de Camargo, Lesley Pritchard-Wiart, Holly Duchow, Sylvia Schell, Laura Bernard, Sara Izadi-Najafabadi, Marie-Josée Fortier, Catherine Wilhelmy and Karine Tremblay.

Contributors $\mathrm{CC}$ is the chief investigator of the WECARE Study, while all coauthors (JGZ, MM, TS, MC, TGP, DBM, M-CB, J-PB, MG, GP, KH, MP, TN, JB, MT) made substantial contributions to the design of the study, to drafting or revising the manuscript and to obtaining funding for the study. Specifically, in terms of study conception and design, CC conceived the study and contributed to all aspects of the study design, while the coauthors primarily provided their expertise in paediatric rehabilitation (JGZ, MC, DBM, GP, KH, MP and TN), in the design and management of experimental trials (MM, M-CB, J-PB and JB), in biostatistical methods (TS), in health economics (TGP) and in telehealth (MG and MT). CC, TS, M-CB and JB drafted the initial WECARE protocol manuscript. All authors (CC, JGZ, MM, TS, MC, TGP, DBM, M-CB, J-PB, MG, GP, KH, MP, TN, JB and MT) read and critically revised the entire manuscript, proposed changes to its content and approved the final version of the manuscript.

Funding This protocol and randomised controlled trial were supported by the Canadian Institutes of Health Research's Early Career Investigator Grants in Maternal, Reproductive, Child and Youth Health (grant number: MRC161356) as well as by matching funds from the CHUS Research Centre, the University of Sherbrooke and the Institut Universitaire de Première Ligne en Santé et Services Sociaux. CC is funded by a salary award from the Fonds de recherche du Québec.

Competing interests None declared.

Patient consent for publication Not required.

Provenance and peer review Not commissioned; externally peer-reviewed.

Supplemental material This content has been supplied by the author(s). It has not been vetted by BMJ Publishing Group Limited (BMJ) and may not have been peer-reviewed. Any opinions or recommendations discussed are solely those of the author(s) and are not endorsed by BMJ. BMJ disclaims all liability and responsibility arising from any reliance placed on the content. Where the content includes any translated material, BMJ does not warrant the accuracy and reliability of the translations (including but not limited to local regulations, clinical guidelines, terminology, drug names and drug dosages), and is not responsible for any error and/or omissions arising from translation and adaptation or otherwise.

Open access This is an open access article distributed in accordance with the Creative Commons Attribution Non Commercial (CC BY-NC 4.0) license, which permits others to distribute, remix, adapt, build upon this work non-commercially, and license their derivative works on different terms, provided the original work is properly cited, appropriate credit is given, any changes made indicated, and the use is non-commercial. See: http://creativecommons.org/licenses/by-nc/4.0/. 
ORCID iDs

Chantal Camden http://orcid.org/0000-0002-5503-3403

Jill G Zwicker http://orcid.org/0000-0002-2986-6938

\section{REFERENCES}

1 Harris SR, Mickelson ECR, Zwicker JG. Diagnosis and management of developmental coordination disorder. CMAJ 2015;187:659-65.

2 Berman-Bieler R, Cabra de Mello M, Farkas A. Early childhood development and disability: a discussion paper. WHO Library Cataloguing-in-Publication Data 2012.

3 Statistique Canada. L'Enquête sur la participation et les limitations d'activités de 2006 : rapport analytique. Ottawa: Canada, 2006: 50p.

4 Henderson SE SD, Barnett AL. Movement assessment battery for Children-2. London, UK: Pearson Assessment, 2007.

5 Smits-Engelsman BCM, Blank R, van der Kaay A-C, et al. Efficacy of interventions to improve motor performance in children with developmental coordination disorder: a combined systematic review and meta-analysis. Dev Med Child Neurol 2013;55:229-37.

6 Peacock-Chambers E, Ivy K, Bair-Merritt M. Primary care interventions for early childhood development: a systematic review. Pediatrics 2017;140:e20171661.

7 Novak I, Mclntyre S, Morgan C, et al. A systematic review of interventions for children with cerebral palsy: state of the evidence. Dev Med Child Neurol 2013;55:885-910.

8 Larroque B, Ancel P-Y, Marret S, et al. Neurodevelopmental disabilities and special care of 5-year-old children born before 33 weeks of gestation (the EPIPAGE study): a longitudinal cohort study. Lancet 2008;371:813-20.

9 Miller AR, Armstrong RW, Mâsse LC, et al. Waiting for child developmental and rehabilitation services: an overview of issues and needs. Dev Med Child Neurol 2008;50:815-21.

10 Missiuna CPH, Pollock N. Strategic management of children with developmental coordination disorder. in: developmental coordination disorder and its consequences. University of Toronto Press, 2015

11 American Psychiatric Association. Diagnostic and statistical manual of mental disorders. 5th ed. Arlington, VA: American Psychiatric Association, 2013

12 Blauw-Hospers $\mathrm{CH}$, Hadders-Algra M. A systematic review of the effects of early intervention on motor development. Dev Med Child Neurol 2005;47:421-32.

13 Winchester SB, Sullivan MC, Marks AK, et al. Academic, social, and behavioral outcomes at age 12 of infants born preterm. West $J$ Nurs Res 2009;31:853-71.

14 Missiuna C, Cairney J, Pollock N, et al. Psychological distress in children with developmental coordination disorder and attentiondeficit hyperactivity disorder. Res Dev Disabil 2014;35:1198-207.

15 Stephenson EA, Chesson RA. 'Always the guiding hand': parents accounts of the long-term implications of developmental coordination disorder for their children and families. Child Care Health Dev 2008;34:335-43.

16 Isa SNI, Ishak I, Ab Rahman A, et al. Health and quality of life among the caregivers of children with disabilities: a review of literature. Asian $J$ Psychiatr 2016;23:71-7.

17 Alonso Soriano C, Hill EL, Crane L. Surveying parental experiences of receiving a diagnosis of developmental coordination disorder (DCD). Res Dev Disabil 2015;43-44:11-20.

18 Missiuna C, Moll S, Law M, et al. Mysteries and mazes: parents' experiences of children with developmental coordination disorder. Can J Occup Ther 2006;73:7-17.

19 Krahn GL, Walker DK, Correa-De-Araujo R. Persons with disabilities as an unrecognized health disparity population. Am J Public Health 2015;105 Suppl 2:S198-206.

20 Morgan C, Novak I, Dale RC, et al. Single blind randomised controlled trial of GAME (Goals - Activity - Motor Enrichment) in infants at high risk of cerebral palsy. Res Dev Disabil 2016;55:256-67.

21 Spittle AJ, Barton S, Treyvaud K, et al. School-Age outcomes of early intervention for preterm infants and their parents: a randomized trial. Pediatrics 2016;138. doi:10.1542/peds.2016-1363. [Epub ahead of print: 22112016$]$

22 Spittle A, Orton J, Anderson PJ, et al. Early developmental intervention programmes provided post hospital discharge to prevent motor and cognitive impairment in preterm infants. Cochrane Database Syst Rev 2015;11:CD005495.

23 Ziviani J, Feeney R, Rodger S, et al. Systematic review of early intervention programmes for children from birth to nine years who have a physical disability. Aust Occup Ther J 2010;57:210-23.
24 McConachie H, Diggle T. Parent implemented early intervention for young children with autism spectrum disorder: a systematic review. $J$ Eval Clin Pract 2007;13:120-9.

25 Simeonsson RJ, Cooper DH, Scheiner AP. A review and analysis of the effectiveness of early intervention programs. Pediatrics 1982;69:635-41.

26 Guralnick MJ. Effectiveness of early intervention for vulnerable children: a developmental perspective. Am J Ment Retard 1998;102:319-45.

27 Palisano RJ, Murr S. Intensity of therapy services: what are the considerations? Phys Occup Ther Pediatr 2009;29:107-12.

28 Rosenbaum P, Gorter JW. The 'F-words' in childhood disability: I swear this is how we should think! Child Care Health Dev 2012;38:457-63.

29 Camden C, Pratte G, Fallon F, et al. Diversity of practices in telerehabilitation for children with disabilities and effective intervention characteristics: results from a systematic review. Disabil Rehabil 2020;42:3424-36.

30 Edirippulige S, Reyno J, Armfield NR, et al. Availability, spatial accessibility, utilisation and the role of telehealth for multi-disciplinary paediatric cerebral palsy services in Queensland. $J$ Telemed Telecare 2016;22:391-6.

31 lacono T, Stagg K, Pearce N, et al. A scoping review of Australian allied health research in ehealth. BMC Health Serv Res 2016;16:543.

32 Camden C, Couture M, Pratte G, et al. Recruitment, use, and satisfaction with a web platform supporting families of children with suspected or diagnosed developmental coordination disorder: a randomized feasibility trial. Dev Neurorehabil 2019;22:470-8.

33 Pratte G, Couture M, Morin M, et al. Evaluation of a web platform aiming to support parents having a child with developmental coordination disorder: brief report. Dev Neurorehabil 2020;23:64-7.

34 Creswell JW, Clark P, Vicki L. Handbook of Mixed Methods in Social \& Behavioral Research. Sage Publications, Inc, 2003.

35 Schulz KF, Altman DG, Moher D. Consort 2010 statement: updated guidelines for reporting parallel group randomised trials. J Pharmacol Pharmacother 2010;1:100-7.

36 Ford I, Norrie J. Pragmatic trials. N Engl J Med 2016;375:454-63.

37 Salisbury C, Thomas C, O'Cathain A, et al. Telehealth in chronic disease: mixed-methods study to develop the tech conceptual model for intervention design and evaluation. BMJ Open 2015:5:e006448.

38 King GA, Tucker MA, Baldwin PJ, et al. Bringing the life needs model to life: implementing a service delivery model for pediatric rehabilitation. Phys Occup Ther Pediatr 2006;26:43-70.

39 Reilly C. Transdisciplinary approach: an atypical strategy for improving outcomes in rehabilitative and long-term acute care settings. Rehabil Nurs 2001;26:244:216-20.

40 Missiuna CA, Pollock NA, Levac DE, et al. Partnering for change: an innovative school-based occupational therapy service delivery mode for children with developmental coordination disorder. Can J Occup Ther 2012;79:41-50.

41 Graham F, Rodger S, Ziviani J. Coaching parents to enable children's participation: an approach for working with parents and their children. Aust Occup Ther J 2009:56:16-23.

42 Kelders SM, Kok RN, Ossebaard HC, et al. Persuasive system design does matter: a systematic review of adherence to web-based interventions. J Med Internet Res 2012;14:e152.

43 Morrison LG. Theory-based strategies for enhancing the impact and usage of digital health behaviour change interventions: a review. Digit Health 2015;1:205520761559533-10.

44 Wilson BN, Crawford SG, Green D, et al. Psychometric properties of the revised developmental coordination disorder questionnaire. Phys Occup Ther Pediatr 2009;29:182-202.

45 Law M, Baptiste S, Carswell A. Canadian Occupational Performance Measure. 5th ed. Ottawa: CAOT PUBLICATIONS ACE, 2014.

46 Verkerk GJQ, Wolf MJMAG, Louwers AM, et al. The reproducibility and validity of the Canadian occupational performance measure in parents of children with disabilities. Clin Rehabil 2006;20:980-8.

47 Cusick A, Lannin NA, Lowe K. Adapting the Canadian occupational performance measure for use in a paediatric clinical trial. Disabil Rehabil 2007;29:761-6.

48 Carswell A, McColl MA, Baptiste S, et al. The Canadian occupational performance measure: a research and clinical literature review. Can J Occup Ther 2004;71:210-22.

49 Kaiser M-L, Braun M, Rhyner C. [Utilization of the Canadian Occupational Performance Measure (COPM) among children and their parents: a Swiss experience]. Can J Occup Ther 2005;72:30-6.

50 James S, Ziviani J, Ware RS, et al. Randomized controlled trial of web-based multimodal therapy for unilateral cerebral palsy to improve occupational performance. Dev Med Child Neurol 2015;57:530-8. 
51 Haley SM, Coster WJ, Dumas HM. Pediatric Evaluation of Disability Inventory Computer Adaptive Test: Development, Standardization and. Boston: Administration Manual, 2012.

52 Haley SM, Coster WJ, Dumas HM, et al. Accuracy and precision of the pediatric evaluation of disability inventory computer-adaptive tests (PEDI-CAT). Dev Med Child Neurol 2011;53:1100-6.

53 Dumas HM, Fragala-Pinkham MA, Haley SM, et al. Computer adaptive test performance in children with and without disabilities: prospective field study of the PEDI-CAT. Disabil Rehabil 2012;34:54:393-401.

54 Pratte G, Schuster T, Berbari J, et al. Parental management of developmental coordination disorder: validation of a French translation of the parental knowledge and skills questionnaire. International Journal of Healthcare 2019;5:36-43.

55 Saigal S, Rosenbaum P, Stoskopf B, et al. Development, reliability and validity of a new measure of overall health for pre-school children. Qual Life Res 2005;14:243-57.

56 Mulhern BJ, Bansback N, Norman R, et al. Valuing the SF-6Dv2 classification system in the United Kingdom using a Discrete-choice experiment with duration. Med Care 2020;58:566-73.

57 Kuhlthau K, Payakachat N, Delahaye J, et al. Quality of life for parents of children with autism spectrum disorders. Res Autism Spectr Disord 2014:8:1339-50.

58 Berry JO, Jones WH. The parental stress scale: initial psychometric evidence. J Soc Pers Relat 1995;12:463-72.

59 Dunst CJ, Trivette CM, Hamby DW. Technical manual for measuring and evaluating family support program quality and benefits. Asheville, NC: Winterberry Press, 2006.
60 Gardner W, Mulvey EP, Shaw EC. Regression analyses of counts and rates: Poisson, overdispersed Poisson, and negative binomial models. Psychol Bull 1995;118:392-404.

61 Zeileis A, Kleiber C, Jackman S. Regression Models for Count Data in R. J Stat Softw 2008;27:1-25.

62 Miles MB HA. Qualitative data analysis - An expanded sourcebook. 2nd ed. Thousand Oaks: International Educational and Professional Publisher, SAGE Publications, Inc, 1994.

63 Ramsey S, Willke R, Briggs A, et al. Good research practices for cost-effectiveness analysis alongside clinical trials: the ISPOR RCTCEA Task force report. Value Health 2005;8:521-33.

64 Guidelines for the economic evaluation of health technologies: Canada. Vol 4th edition. Ottawa: Canadian agency for drugs and technologies in health, 2017

65 Burton A, Billingham LJ, Bryan S. Cost-Effectiveness in clinical trials: using multiple imputation to deal with incomplete cost data. Clin Trials 2007;4:154-61.

66 Black WC. The Ce plane: a graphic representation of costeffectiveness. Med Decis Making 1990;10:212-4.

67 Hoch JS, Rockx MA, Krahn AD. Using the net benefit regression framework to construct cost-effectiveness acceptability curves: an example using data from a trial of external loop recorders versus Holter monitoring for ambulatory monitoring of "community acquired" syncope. BMC Health Serv Res 2006;6:68.

68 Fenwick E, O'Brien BJ, Briggs A. Cost-effectiveness acceptability curves-facts, fallacies and frequently asked questions. Health Econ 2004;13:405-15. 